\title{
Effective Medium Theory of the Optical Properties of Aligned Carbon Nanotubes
}

\author{
F.J. García-Vidalit, J.M. Pitarke目 and J.B. Pendry \\ Condensed Matter Theory Group, \\ The Blackett Laboratory, Imperial College, \\ London $S W^{\text {r }} 2 B Z(U K)$.
}

We present an effective medium theory in order to analyze the reported optical properties of aligned carbon nanotube films (W.A. de Heer et al., Science 268, 845 (1995)). This methodology is based on photonic band structure calculations and allows treatment of complex media consisting of particles that interact strongly. We also develop a simple Maxwell-Garnett type approach for studying this system. In comparing the results of both mean field theories, we demonstrate that the inclusion of the full electromagnetic coupling between the nanotubes, as our numerical scheme does, is necessary for a complete explanation of the experimental data.

\section{PACS numbers: 78.66.Sq, 41.20.Jb, 61.46.+w, 73.20.Mf}

Since the discovery of tubular fullerenes in 1991 [1], several experimental and theoretical studies of the unique properties of these materials have been carried out. Despite the short time since the first carbon nanotube was synthesised, possible applications of these new materials have already been reported. Nanotubes can be used as atomic-scale field emitters [2] or as a pinning material in high- $T_{c}$ superconductors [3] whereas it has been proposed that hollow tubes might serve as nanoscale molds. The unique properties of these carbon tubes are derived from their special topology and their nanometric dimensions. Very recent measures of the conductivity of individual nanotubes 44 suggest that the geometry of these tubes plays a fundamental role in determining if the nanotube is metallic or semiconducting. From the mechanical point of view, nanotubes seem to be very strong and flexible at the same time due to the ability of $\mathrm{sp}^{2}$ carbon atoms to rehybridize when the bonds are deformed.

These carbon nanotubes also present very interesting optical properties. Thin films of aligned carbon tubes are birefringent, reflecting differences in the dielectric function for light polarized along (s-polarization) and normal (p-polarization) to the tubes [5]. Up to our knowledge, a complete theoretical analysis of the optical properties of these arrays of carbon nanotubes is still missing.

In analyzing the propagation of electromagnetic waves in heterogeneous media, various effective medium approaches like the Maxwell-Garnett (MG) approximation [6] have been used to determine the effective dielectric constant of different composite structures. Very recently, the validity of the MG approach has been checked by comparing their results for periodic dielectric systems with the ones obtained in the long-wavelength limit of photonic band structure calculations [7]. For these structures, the MG results seem to agree reasonably well with their vector-wave counterparts for small filling ratios, but in the close-packing regime the inclusion of the full electromagnetic (EM) interaction between the different objects is unavoidable. Only numerical methods give the capacity to treat this more complex limit.

Our idea is to apply a numerical methodology originally constructed to study photonic materials and the propagation of EM waves in complex media [\$] to analyze the optical properties of these carbon nanotube films where the tubes form a very close-packed structure. Within this framework it is possible to develop an effective medium theory in which the EM interaction between the constituents of the complex system is fully included [9]. We also want to develop a MG-like approach able to study arrays of carbon nanotubes and compare these results with our exact results, studying the range of validity of MG approximation for carbon based materials.

Our model for a carbon nanotube film is shown in figure 1a. An array of tubes, infinitely long in the $z$ direction, is arranged on a square lattice. The diameter of the cylinders is chosen to be $10 \mathrm{~nm}$, in accordance with experimental evidence [5]. The distance between the nanotubes, $d$, will be varied throughout our calculations, studying the dependence of the optical properties of this structure on the separation between the tubes.

Let us discuss first the dielectric model we are using for describing the dielectric characteristics of an isolated carbon nanotube. Carbon nanotubes are made of a number of cylindrical shells of planar graphite. Graphite is highly anisotropic and its optical response depends on the direction of the electric field with respect to the axis normal to the graphite sheets. Therefore, for a given frequency $\omega$, it is necessary to distinguish two different components, $\epsilon_{\|}(\omega)$ and $\epsilon_{\perp}(\omega)$, in its dielectric function for the directions parallel and normal to the axis, respectively. These components have been measured by several methods, leading to slightly different sets of data. For our calculations we have used the dielectric functions of graphite as tabulated in Ref. 10. In transfering these dielectric constants to cylindrical multishells we will assume that this material is a dielectric continuum and locally identical to graphite. A similar transfer procedure has been previously applied to compute the UV absorption of multishell fullerenes [11]. At every point inside the nanotube we can write down a local dielectric tensor that in cylindrical coordinates (see fig 1b) takes the 
diagonal form:

$$
\hat{\varepsilon}(\omega)=\varepsilon_{\perp}(\omega)(\theta \theta+\mathbf{z z})+\varepsilon_{\|}(\omega) \mathbf{r r} .
$$

In Cartesian coordinates, the tensor like character of the dielectric function is more pronounced:

$$
\hat{\varepsilon}(x, y, \omega)=\left(\begin{array}{ccc}
\frac{x^{2}}{r^{2}} \varepsilon_{\|}+\frac{y^{2}}{r^{2}} \varepsilon_{\perp} & \frac{x y}{r^{2}}\left(\varepsilon_{\|}-\varepsilon_{\perp}\right) & 0 \\
\frac{x y}{r^{2}}\left(\varepsilon_{\|}-\varepsilon_{\perp}\right) & \frac{y^{2}}{r^{2}} \varepsilon_{\|}+\frac{x^{2}}{r^{2}} \varepsilon_{\perp} & 0 \\
0 & 0 & \varepsilon_{\perp}
\end{array}\right) .
$$

Arc-generated multilayered nanotubes are generally hollow. Their inner radius distribution usually varies from 0.25 to $2 \mathrm{~nm}$, being tubes with inner radius of 0.75 to $1 \mathrm{~nm}$ the most abundant ones in the samples [12]. The fundamental importance of the hollow character of isolated nanotubes in their dielectric response has been previously stressed 113. However we have found that when nanotubes form close-packed structures, as in nanotube films, the presence of a hollow core plays a much less important role. Moreover, our results indicate that in the strong coupling limit and for p-polarized light, the hollow core can be safely ignored if the ratio between the inner and the outer radii of the tubes is less than 0.4. As the nanotubes present in the experiment [5] seem to fulfill this condition, for p-polarized light we will only show results for plain cylinders (inner radius equal to zero). A detailed discussion of the effect of a hollow core on the dielectric properties of aligned carbon nanotubes will be presented elsewhere [14].

If the nanostructured film could be replaced by an effective homogeneous medium, a single pair of Bloch waves for each polarization, $\left(k_{s}(\omega),-k_{s}(\omega)\right)$ and $\left(k_{p}(\omega)\right.$,$k_{p}(\omega)$ ), should dominate the photonic band structure of the system. It is indeed the case that over the entire range of frequencies $\omega$, four EM waves are dominant and we can use the dispersion relation of these Bloch waves to define the effective dielectric constants for $\mathrm{s}$ and $\mathrm{p}$ polarizations

$$
\varepsilon_{e f f}^{s}(\omega)=\frac{c^{2} k_{s}^{2}(\omega)}{\omega^{2}}, \quad \varepsilon_{e f f}^{p}(\omega)=\frac{c^{2} k_{p}^{2}(\omega)}{\omega^{2}},
$$

where $c$ is the speed of light.

In order to calculate the photonic band structure of the array of carbon nanotubes shown in figure 1a, we need to find solutions of the Maxwell equations with a tensor dielectric function,

$$
\begin{aligned}
\nabla \times \mathbf{E} & =-\mu_{0} \mu \partial \mathbf{H} / \partial t, \\
\nabla \times \mathbf{H} & =+\varepsilon_{0} \hat{\varepsilon}(\mathbf{r}, \omega) \partial \mathbf{E} / \partial t,
\end{aligned}
$$

that obey Bloch's theorem. On-shell methods like the one described in Ref.[8] are ideally suited to calculate the band structure of materials whose dielectric function depends on $\omega$, as graphite does. In these methods, $\omega$ is fixed at first and hence $\varepsilon(\omega)$ can be specified, allowing us to establish an eigenvalue equation for the calculation of $k(\omega)$. In particular this approach has a great advantage for studying EM waves in metals [15] where the dielectric function depends strongly on frequency. Very recently [16], an extension of this formalism has been developed which is able to work with tensor like dielectric functions as the ones present in eqs. (4). Details of this formalism can be found in Refs. [8] and [16].

With this methodology we calculate all the allowed Bloch waves of our system for a given photon energy $\omega$. Then we can extract the dominant ones looking at the imaginary parts of their wavevectors. Finally, we can define the effective dielectric constants using Eq. (3).

In its generalized version, MG approach is just the application of Clausius-Mossotti relations to describe the dielectric properties of composite materials in an effective way. The basic assumption of this approach is that the polarizability of an individual object is modified by the presence of another ones only via their depolarizing contribution to the electric field acting on the object. For the case of arrays of cylinders in vacuum, a MG effective dielectric constant can be written as a function of the polarizability of an individual tube $\alpha_{\sigma}(\omega)$, and a depolarization factor $L_{\sigma}$, which both depend on the polarization of the incident light, $\sigma$ :

$$
\varepsilon_{e f f}^{\sigma, M G}(\omega)=1+\frac{f \alpha_{\sigma}(\omega)}{1-f L_{\sigma} \alpha_{\sigma}(\omega)},
$$

where $f$ is the volume fraction occupied by the cylinders.

For p-polarized light $L_{p}=\frac{1}{2}$ and the polarizability of an individual plain cylinder is [13] :

$$
\alpha_{p}(\omega)=2 \frac{\varepsilon_{\|}(\omega)-\Delta}{\varepsilon_{\|}(\omega)+\Delta},
$$

where $\Delta=\sqrt{\frac{\varepsilon_{\|}(\omega)}{\varepsilon_{\perp}(\omega)}}$. Introducing this expression into Eq.(5), the effective dielectric constant for p-polarization can be written as:

$$
\varepsilon_{e f f}^{p, M G}(\omega)=\frac{\varepsilon_{\|}(\omega)+\Delta+f\left(\varepsilon_{\|}(\omega)-\Delta\right)}{\varepsilon_{\|}(\omega)+\Delta-f\left(\varepsilon_{\|}(\omega)-\Delta\right)} .
$$

For s-polarized light, $L_{s}=0$ and the polarizability of a plain tube is just the same as the polarizability of the homogeneous medium, $\alpha_{s}(\omega)=\varepsilon_{\perp}(\omega)-1$, leading to a very simple formula for the effective dielectric constant:

$$
\varepsilon_{\text {eff }}^{s}(\omega)=f \varepsilon_{\perp}(\omega)+(1-f) .
$$

If we wish to consider the general case of hollow cylinders, $f$ must be replaced in eq. (8) by $f^{\prime}=f\left(1-\gamma^{2}\right), \gamma$ being the ratio between the inner and outer radii of the tubes.

Eq.(8) is the exact result for the effective dielectric constant for s-polarization. For this polarization there is no EM interaction between the tubes as MG approach assumes $\left(L_{s}=0\right)$ and the total polarizability is just the 
sum of the polarizabilities of the individual nanotubes. Because the electric field is directed along the tubes, the optical response of the system depends only on $\varepsilon_{\perp}(\omega)$. Our numerical results for this polarization are in complete agreement with Eq.(8), giving additional support to our scheme.

The simple relation (Eq.(8)) that holds for spolarization can serve as an indirect way to estimate the volume fraction occupied by the nanotubes, $f$. The imaginary part of $\varepsilon_{\text {eff }}^{s}$ is simply $f^{\prime}$ multiplied by $\operatorname{Im} \varepsilon_{\perp}$. This is the reason why the experimental $\operatorname{Im} \varepsilon_{\text {eff }}^{s}$ [5] has its maximum at $4.6 \mathrm{eV}$ just as $\operatorname{Im} \varepsilon_{\perp}$ does $[10$. Then, a rough estimation of $f$ can be derived using the different heights of the peaks at $4.6 \mathrm{eV}$ for the reported $\operatorname{Im} \varepsilon_{\text {eff }}^{s}$ and the experimental $\operatorname{Im} \varepsilon_{\perp}$. Taking the inner radius of the tubes to be $0.25-2 \mathrm{~nm}$, we find $f \approx 0.6-0.7$.

For p-polarized light, the EM interaction between the nanotubes is not negligible and our numerical method can give new insights in the analysis of this interaction. In figures 2 and 3 we show our numerical results for the effective dielectric constant, comparing them with the results of the MG-like approach given by Eq.(7).

With figure 2 our aim is to analyze the behaviour of these dielectric constants for low volume fractions, $f$ varying from $f=0.09(d=30 \mathrm{~nm})$ to $f=0.27$ $(d=17 \mathrm{~nm})$. As we can see from this figure, the good agreement between MG results and our method is remarkable for these values of $f$; only at low frequencies there are some small differences in $\operatorname{Im} \varepsilon_{\text {eff }}^{p}$ between both mean field theories. It is known that $\mathrm{MG}$ approach works very well in the dilute limit for dielectric media [7], and our numerical results show this is also the case for aligned carbon nanotubes. As we can conclude by looking at the two peaks present in $\operatorname{Im} \varepsilon_{\text {eff }}^{p}(\omega)$, the optical response of the structure in the dilute limit is governed by the dipolar EM modes of an isolated carbon nanotube. Due to the anisotropy of the dielectric function of graphite, there are two dipolar modes, one located energetically at 5.3 $\mathrm{eV}$ and the other at a higher energy, $6.3 \mathrm{eV}$.

In figure 3 we study the case in which the EM interaction between the nanotubes becomes important, showing our results for the effective dielectric constant for distances between the tubes smaller than three times their radii $(f>0.3)$. In the same figure we show the results of Eq. (7) for this range of distances and the experimental data for p-polarization as reported in Ref. [5]. We can see that the effect of the EM interaction is basically to shift to lower energies the low energy dipolar mode (from 5.3 $\mathrm{eV}$ for isolated nanotubes to $4.8 \mathrm{eV}$ for very close contact nanotubes), and conceal the dipolar mode at $6.3 \mathrm{eV}$. Again, MG approach seems to be a very good approximation for $\varepsilon_{e f f}^{p}(\omega)$ for intermediate distances $(d>11.5 \mathrm{~nm})$ revealing that, for this range of distances, the EM coupling between the tubes is basically a dipolar one as MG assumes. However, the inclusion of the full EM interaction between the tubes as our mean field theory does is necessary in order to describe properly the strong coupling limit $(d \leq 11.5 \mathrm{~nm})$. The experimental imaginary part of the effective dielectric constant presents its maximum just at $4.8 \mathrm{eV}$, and our results suggest that the position of this peak can only be explained if the nanotubes are indeed in very close contact $(d \approx 10.3 \mathrm{~nm})$ forming the films. As regards to the real part of $\varepsilon_{\text {eff }}^{p}$, the shape of the theoretical curve for $d=10.3 \mathrm{~nm}$ closely matches the experimental data but a constant shift of approximately 1 is needed in order to obtain an excellent agreement between theory and experiment.

In conclusion, we have presented an effective medium approach to analyze the optical properties of carbon nanotube films. In contrast with usual effective medium methods, our numerical approach can treat the limit in which the EM interaction between the constituents of a complex system is important, as well as the dilute limit. We have also developed a Maxwell-Garnett approach to study this system. Using both mean field theories we have analyzed in detail the EM interaction between the nanotubes as a function of the volume fraction. MG approach appears to be a good approximation for large and intermediate distances between the tubes. However, the inclusion of the full EM coupling between the nanotubes is essential in order to study closed-packed structures, appearing in aligned carbon nanotube films [5]. The good agreement obtained between our numerical results and the experimental data demonstrates that our mean field theory can be a very useful tool to analyze spectroscopic properties of nanostructured materials.

One of us (FJGV) acknowledges financial support of the Human Capital and Mobility Programme of the European Union under contract ERBFMBICT 950397 and useful discussions with Carmina Monreal and Fernando Flores. JMP gratefully acknowledges financial support of the University of Basque Country, the Spanish Comisión Asesora Científica y Técnica (CICYT) and the Basque Unibertsitate eta Ikerketa Saila. Part of this work was supported by EU contract No. ERBCHRXCT 930342.

$\dagger$ Present address: Departamento de Física Teórica de la Materia Condensada. Facultad de Ciencias. Universidad Autónoma de Madrid. Madrid 28049 (Spain).

¥ Permanent address: Materia Kondentsatuaren Fisika Saila. Zientzi Fakultatea. Euskal Herriko Unibersitatea. 48080 Bilbo (Spain).

$1 \quad$ S. Iijima, Nature 354, 56 (1991).

$2 \quad$ A.G. Rinzler et al., Science 269, 1550 (1995); W.A. De Heer, A. Chatelain and D. Ugarte, Science 270, 1179 (1995).

3 K. Fossheim et al., Physica C 248, 63 (1995).

4 L. Langer et al., Phys. Rev. Lett. 76, 479 (1996); T.W. Ebbesen et al., Nature 382, 54 (1996).

$5 \quad$ W.A. De Heer et al., Science 268, 845 (1995).

6 J.C. Maxwell-Garnett, Phil. Trans. R. Soc. 203, 385 
(1904); D.M. Wood and N.W. Ashcroft, Phil. Mag. 35, 269 (1977).

7 S. Datta et al., Phys. Rev B 48, 14936 (1993); R. Tao, Z. Chen and P. Sheng, Phys. Rev. B 41, 2417 (1990).

8 J.B. Pendry and A. MacKinnon, Phys. Rev. Lett. 69, 2772 (1992); J.B. Pendry, J. Mod. Opt. 41, 209 (1994).

9 J.B. Pendry and L. Martín Moreno, Phys. Rev. B. 50, 5062 (1994).

10 Handbook of Optical Constants of Solids, edited by E.D. Palik (Academic, Orlando) (1985).

11 A.A. Lucas, L. Henrard and Ph. Lambin, Phys. Rev B 49, 2888 (1994).

12 D. Ugarte, A. Chatelain and W.A. de Heer, Science 274, 1897 (1996).

13 L. Henrard and Ph. Lambin, J. Phys. B 29, 5127 (1996);

L. Henrard et al., Full. Sci and Tech. 4, 131 (1996).

14 J.M. Pitarke, F.J. García-Vidal and J.B. Pendry, to be published.

15 F.J. García-Vidal and J.B. Pendry, Phys. Rev. Lett. 65, 3152 (1996); F.J. García-Vidal and J.B. Pendry, Prog. Surf. Sci. 50, 55 (1995).

16 A.J. Ward and J.B. Pendry, J. Mod. Opt. 43, 773 (1996).

\section{Figure Captions}

Figure 1. a) Our model of a carbon nanotube film: an array of cylinders, infinitely long in the $z$-direction, is arranged on a square lattice in the xy-plane. b) Unit cell of the structure, $R=5 \mathrm{~nm}$ is the radius of the cylinders, $d$ is the distance between the centers of the tubes and $L_{y}$ is the thickness in the direction of propagation of the light that will be chosen equal to $d$. Also shown are the cylindrical unit vectors, $\hat{\theta}$ and $\hat{r}$ in order to describe the change of coordinates from cylindrical to Cartesians and the subsequent change in the tensor like dielectric function associated to a generic point inside the tube $(\mathrm{x}, \mathrm{y})$ (see text).

Figure 2. The real and imaginary parts of the effective dielectric constant for p-polarized light, with $d$ varying from $d=30 \mathrm{~nm}$ to $d=17 \mathrm{~nm}$. Full line: our numerical results. Dashed line: results using a MG approach (see eq.7).

Figure 3. The real and imaginary parts of the effective dielectric constant for p-polarized light for different values of $d$. (a) $d=10.3 \mathrm{~nm}$ (b) $d=10.7 \mathrm{~nm}$ (c) $d=11.5 \mathrm{~nm}$ (d) $d=12.5 \mathrm{~nm}(\mathrm{e}) d=14 \mathrm{~nm}$. Full line: our numerical results. Dashed line: results using a MG approach (see eq.7). The dots represent the experimental data as reported in [5]. For the sake of clarity, the curves for the real part are shifted by a quantity shown in the figure. The experimental values of $R e \varepsilon_{e f f}^{p}$ are shifted by a constant value of +5 with respect to the reported ones. 



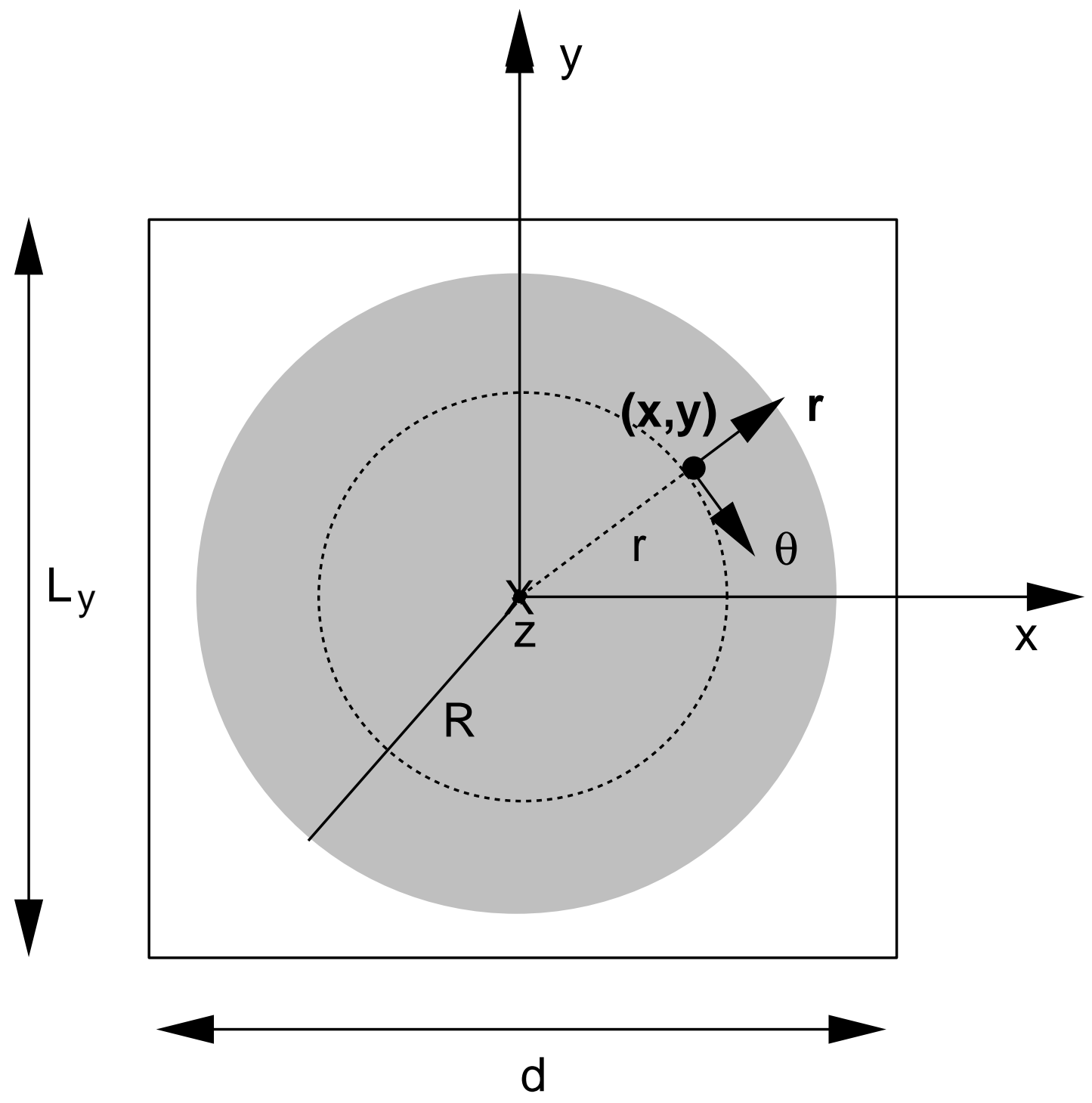




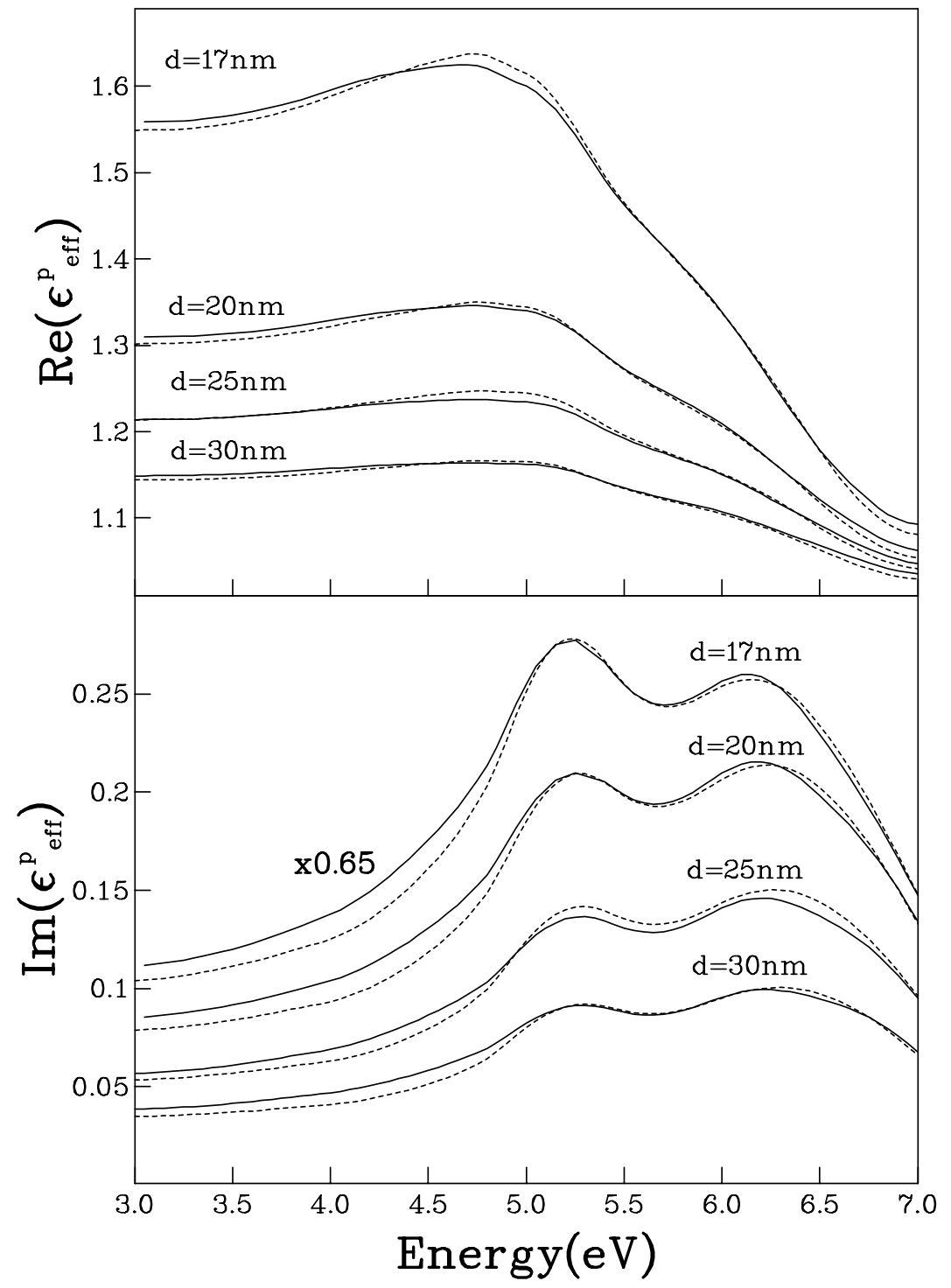




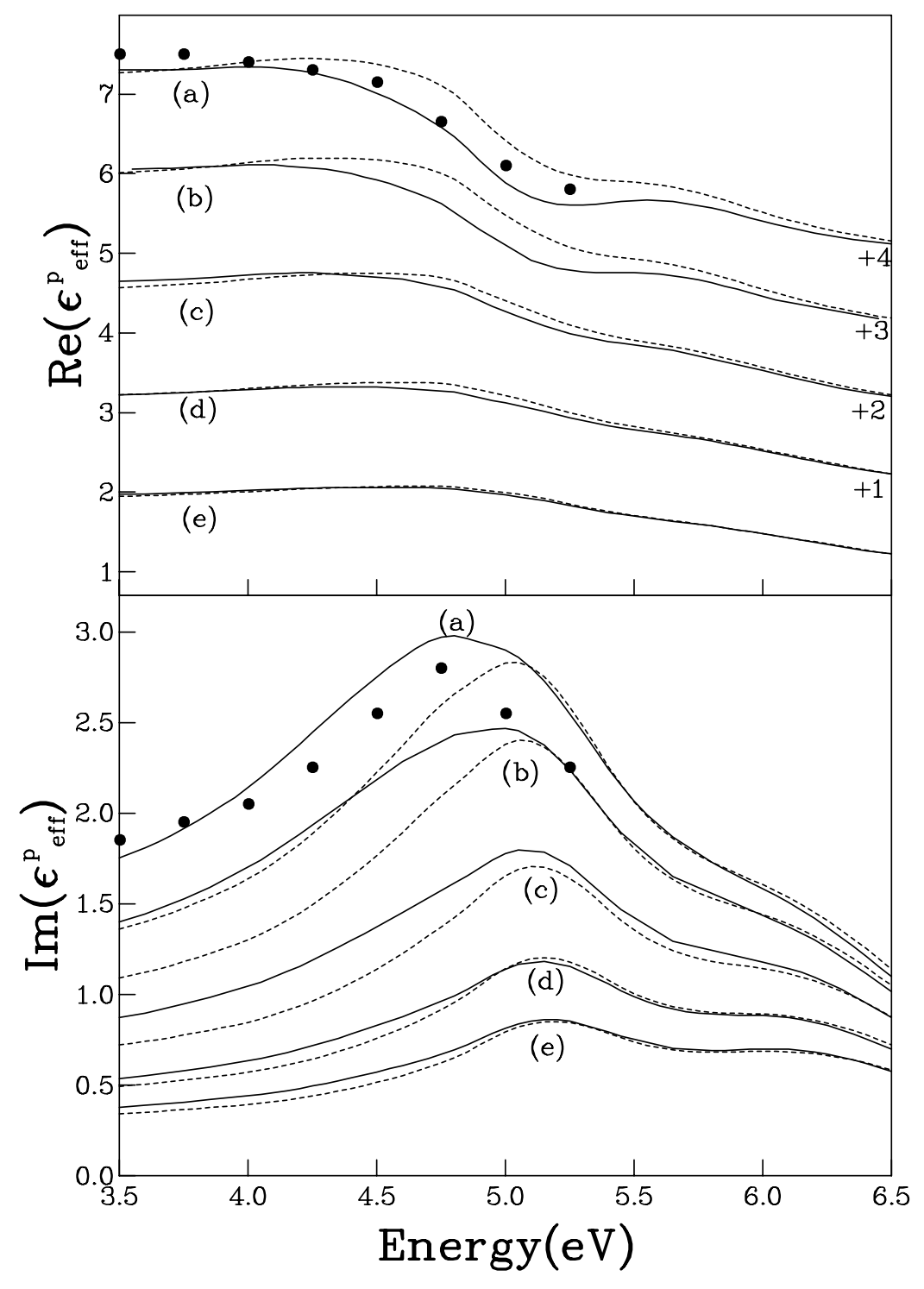

